\title{
REVIEW ON STRUCTURAL HEALTH INTERROGATION USING FIBRE BRAGG GRATING SENSORS
}

\author{
Peteris Skels ${ }^{1}$, Rims Janeliukstis ${ }^{2}$, Viktors Haritonovs ${ }^{1}$ \\ ${ }^{1}$ Riga Technical University, Department of Roads and Bridges, Latvia; \\ ${ }^{2}$ Riga Technical University, Institute of Materials and Structures, Latvia \\ peteris.skels_1@rtu.lv, rims.janeliukstis_1@rtu.lv, viktors.haritonovs@rtu.lv
}

\begin{abstract}
Fibre Bragg Grating sensors are optical fibre sensors that are now widely used in civil, mechanical and aerospace engineering for structural health monitoring and damage detection purposes. Typical parameters to be measured are strain and temperature, but also rotation, pressure, vibrations, the distance between stationary and moving or rotating components and concentration of chemical substances can be registered. These sensors have numerous advantages with respect to their piezoelectric counterparts, such as light weight, small dimensions, immunity to electromagnetic interference caused by nearby electronic devices. One of the most notable advantages of Fibre Bragg Grating sensors is the multiplexing capability, meaning that several tens to hundreds of sensors can be stacked together to increase the number of measurement points. This is a key advantage in structural health monitoring and damage detection in aerospace, pavement, bridge and other structures, where numerous points on the structure are subjected to high stresses. Moreover, fibre optical sensors are fully passive they are capable of functioning without power supply. This review paper deals with highlighting the usage of Fibre Bragg Grating sensors in structural health monitoring and damage detection in various types of structures, namely, civil structures (bridges, pavements) and lightweight structures (aircraft, thin-walled composites), thus underlining a great significance of these sensors in structural safety.
\end{abstract}

Keywords: optical fibre, FBG sensors, damage detection, SHM.

\section{Optical sensors overview}

Optical fibres consist of two concentric silica layers, namely, the core and the cladding. The small differences in refractive index between both layers ensure the condition of total internal reflection of light inside the fibres, making the optical fibres efficient waveguides. Fibre optical sensors (FOS) comprise the core with a diameter of about $8 \mu \mathrm{m}$ made of silica doped with germanium oxide and a cladding layer with a diameter of $125 \mu \mathrm{m}$ made of pure silica. The smallest signal attenuation 0.2 $\mathrm{dB} / \mathrm{km}$ is achieved at the wavelength of $1.550 \mathrm{~nm}[1]$.

The essential difference between conventional and fibre optical sensors (FOS) is that FOS are passive - they are capable of fully functioning without power supply. Providing electrical power to the sensor in remote areas for road pavements, bridges or aircraft can be a very difficult task. FOS are sensitive to temperature, pressure, strain, gas concentration, vibrations, the distance between stationary and moving or rotating components, etc. and the raw FOS measurements are in a form of wavelength information, which is analysed and processedto reveal the vital information about the health state of the structure [2]. Optical sensors also provide higher accuracy compared to electronic analogues. This is due to the fact that the light wavelength is much shorter $(1.3-1.5 \mu \mathrm{m})$ than the radio waves used in wireless systems (in $\mathrm{GHz}$, wavelength measured in centimetres).

The most promising type of fibre optic sensors used in sensor applications is optical sensors based on fibre Bragg grating (FBG). FBG sensors have several advantages over electric metallic sensors [3; 4]:

- compatible with various composite materials, such as reinforced glass or carbon fibre reinforced composites, FBG can be embedded in the composite material itself or fixed on the surface of the test object;

- FBG can be used for measuring large deformations $\left(>10000 \mu \mathrm{m} \cdot \mathrm{m}^{-1}\right)$ and are suitable for high load-bearing structures;

- FBG sensors are light and compact, small size and weight;

- FBG is immune to external electromagnetic effects;

- FBG is essentially a passive (non-electric) element. Consequently, it can be located near high voltage and at high fire hazard points;

- FBG signals can be transmitted over long distances ( $>20 \mathrm{~km}$ depending on the type of fibre used and the light source); 
- FBG sensors can be connected to a single optical fibre (depending on the applied signal processing method up to 100 and more);

- Significant long-term stability of the parameters;

- Easy maintenance and operation of sensors;

- Very good corrosion resistance;

- Special FBG sensors can be used at very high temperatures $\left(>700{ }^{\circ} \mathrm{C}\right)$;

- Relatively simple and quick to install;

- Passive optical sensors alone do not have a meaningful value, so reducing the risk of theft.

Shortcoming of FBG optical sensors is that the optical fibre is brittle. Bare FBGs are extremely fragile and can be easily damaged during their service life. Hence, suitable packaging methods are crucial. FBG sensors can be encapsulated in either metal slice using glue/ epoxy resin or fibre reinforced polymer bar to monitor metal and concrete structures. Another common packaging is a container (tube or groove) [3].

Fibre optical sensors (FOS) are divided in two main groups - intrinsic and extrinsic sensors. In case of extrinsic, the optical fibre is used only for transferring the signal to external sensor system, whereas, in case of intrinsic sensors, it is not compulsory for signal to leave the fibre. FBG sensors are intrinsic.

\section{Investigation and analysis of FBG}

Wavelength-modulated spectrometric sensors are based on the reception of optical signal wavelength changes, which are dependent on external variations or distortions. Such sensor types include the fibre Bragg grating (FBG) sensors and fluorescence sensors. The FBG technology is mainly based on a simple design and a relatively strong reflection signal [2].

In comparison to other FOS, the FBG sensor technology offers additional advantages, for example, Fabry-Perot interferometric sensors cannot be efficiently stacked or multiplexed and long gauge FOS are not suitable for registering impact events, which is crucial in safe aircraft service[5]. Typical FBG sensor characteristics are shown in Table 1.

Table 1

Characterization of FBG [5]

\begin{tabular}{|l|l|}
\hline Sensor type & -Point, -Semi-distributed \\
\hline Typical registered quantities & -Temperature, -Strain, -Rotation, -Pressure \\
\hline Multiplexing & -Quasi-distributed, -Wavelength-division \\
\hline Measurement points in 1 line & $10-50$ \\
\hline Typical resolution for strain & $1 \mu \varepsilon$ \\
\hline Typical resolution for temperature & $0.1^{\circ} \mathrm{C}$ \\
\hline Spatial resolution & 0.1 \\
\hline
\end{tabular}

FBG sensors are beneficial also in terms of linearity - wavelength shift due to temperature and axial strain is linear [1].

For optic fibre, which has a FBG grid, the refractive index has a periodic structure. Grid period $\Lambda$, $\mathrm{nm}$ is the distance between two, adjacent peaks of the refractive index [5]. Because of its structure, the FBG reflects the wavelengths of a certain length of light $\lambda_{B}$ from certain optical fibre points, but does not affect the propagation of other wavelengths in the grid. A periodic structure of such a grid is obtained on the base of silicon optical fibre with germanium admixture, in the segment of a small optical fibre core that is irradiated with ultraviolet (UV) light. The wavelength of light that will be reflected in the Barge grid is expressed by the following formula:

$$
\lambda_{B}=2 \Lambda n_{B}
$$

where $\lambda_{B}$ - reflected wave length from certain FBG point, nm;

$\Lambda$ - FBG period, nm;

$n_{B}-$ effective index of refraction. 
The FBG operation scheme is depicted in Figure 1.

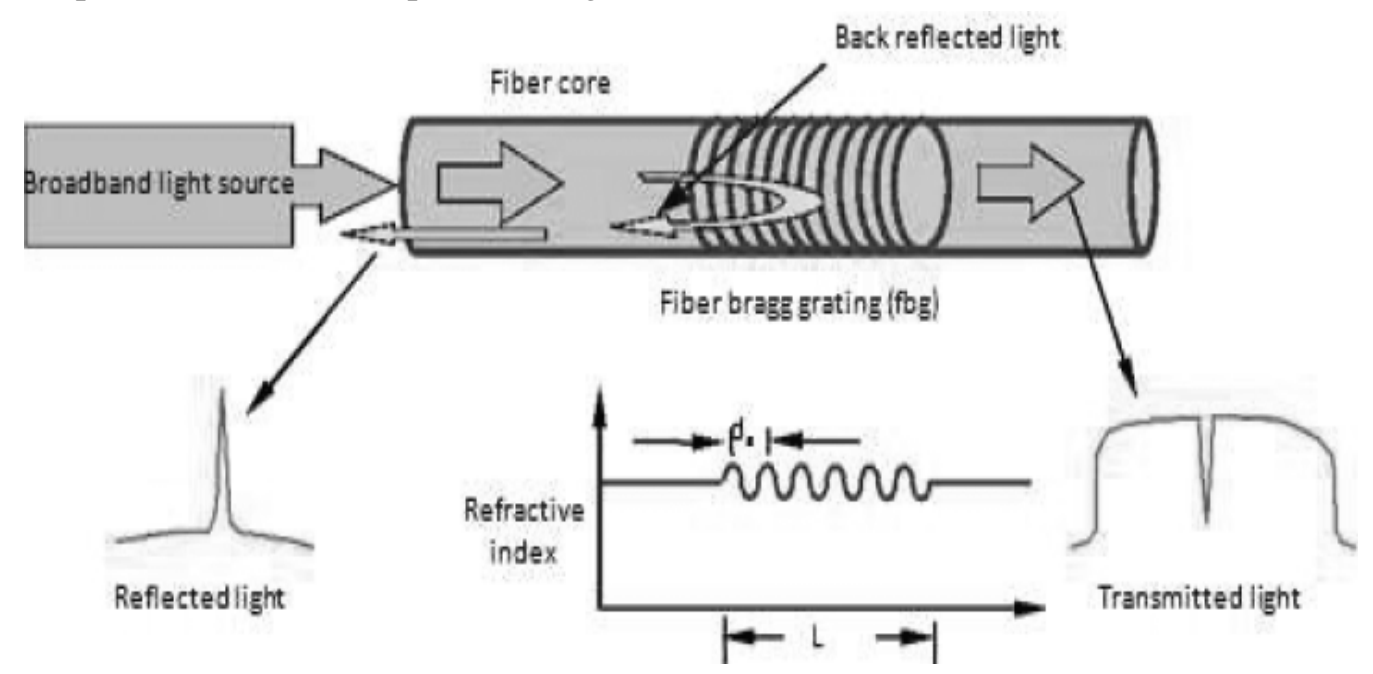

Fig. 1. Operational scheme of Fibre Bragg Gratings [5]

\section{SHM technologies for composite structures}

The manufacturing of smart structures involves the integration of sensors into materials. The FBG sensors are one of the best candidates due to the ease of embedding into composite and concrete and ability to reveal the accumulation of stresses in the process of manufacturing [1].

Multiple FBG sensors can be stacked together or multiplexed having the ability to monitor various parts of the structure.For the application of FBG sensors in SHM, a light source and light detection system are needed to illuminate and interrogate the sensor(s), respectively [4].

\section{Ultrasonics-based damage detection}

Damage monitoring of composite structures is mostly based on guided ultrasonic wave principle. The main drawback of classical ultrasonic structural inspections is the point-by-point scanning of the structure, which is tedious and time-consuming. One major benefit of guided waves is in their rapid global inspection capability, since they propagate over considerable distances [6]. The second one is that ultrasonic wave methods can work without the limitation of the direction of crack [2]. The transmitter (actuator) of guided ultrasonic waves is usually a piezoceramic patch, since they are inexpensive, available in small sizes and can function both, as actuators and sensors [4]. However, piezoceramic transducers and related electrical wiring for data acquisition can be affected by and/or be the source of electromagnetic and radio frequency interference impacting the performance of any electrical systems that are stationed nearby. The registered signal typically is very weak, hence an amplifier has to be set. This again involves more wires and costs [4; 5].FBG sensors are a proper substitute to the conventional piezoceramic sensors $[2 ; 4]$ to register the high frequency waves because no complex wiring is required. These sensors offer an attractive possibility for the full-scale monitoring of structural components thanks to the sensor multiplexing in a single fibre [5].

Lamb waves among other guided ultrasonic waves are intensively exploited in damage detection and identification in composite structures. The advantages of the Lamb wave method are the ability of long-distance transmission and high sensitivity to both the surface and the internal defects [7]. Lamb waves comprise symmetric and asymmetric modes that can propagate independently of the other. The analysis of ultrasonic waves is complex due to the complicated behaviour of waves in the material. This involves reflections of waves from boundaries, absorption and transmission.

The essential part in the analysis of Lamb wave propagation is to build the dispersion curves [6]. These curves depict the phase and group velocities of the waves with respect to the frequency of wave excitation (Figure 2). The dispersive nature of waves means that the different frequency components of the Lamb waves travel at different speeds and that the shape of the wave packet changes as it propagates through solid media $[4 ; 8]$. The dispersion curves can be obtained by solving the wave equation. 
This dispersion curve is used to

- determine a frequency range, where only the fundamental modes $S_{0}$ and $A_{0}$ propagate [4], which is done in order to avoid superposition between the modes that would complicate the extraction of damage information;

- find any non-dispersive regions.

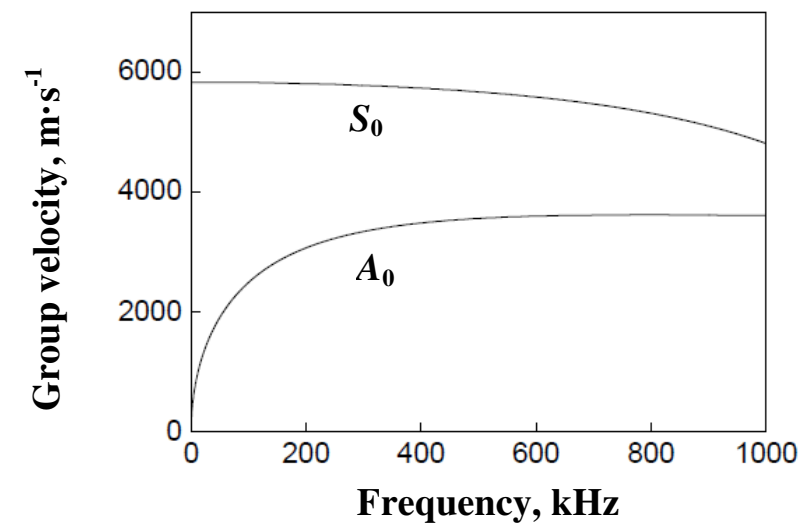

Fig. 2. Dispersion curves for group velocity of symmetric $S_{0}$ and asymmetric $\boldsymbol{A}_{0}$ Lamb modes for CFRP plate [6]

In damage detection problems, the healthy specimen is used as a reference - the time of flight of the wave is measured for a known distance between sensors to obtain the group velocity of the waves. The disturbance caused by the travelling wave has an impact on the grating pitch of the FBG sensor. This phenomenon induces the shift of the Bragg wavelength. One of the main advantages of using exciting Lamb waves for damage monitoring is that there is no need for analytical models conclusions on damage characteristics, such as the coordinates and the size can be drawn by solely analysing the waves in the path between the actuator and sensor [5].

Acoustic waves are usually excited in the form of tone burst signal with a particular frequency deduced from the non-dispersive regions of the dispersion curve plot. Typically, this driving frequency in SHM applications exceeds $50 \mathrm{kHz}$ [5].

In order for the damage identification system to work efficiently, the grating length of FBG sensors should be less than the wavelength of the propagating Lamb wave. An ideal value of the ratio of the wavelength of the Lamb wave to the grating length is set as of 6:1 [5].

\section{Aircraft monitoring}

In an aircraft design, there is a need to replace aluminium with composites for the following 3 reasons: fatigue problem with aluminium, lightness of composites, and corrosion problem in aluminium. The aircraft manufacturers have been using composite materials in various parts of the aircraft [9].

The honeycomb sandwich composite, mainly non-metallic, has been used in larger parts in the aircraft. However, the main problem with sandwich composite is delamination between the core and the facesheet, and this failure occurs underneath the skin and cannot be seen with naked eye [9]. Thus, an efficient cost-saving monitoring system is needed for early damage detection.

When it comes to aircraft monitoring, two stages can be discerned - the measurement of local stress concentrations in the case of fatigue issues (also known as monitoring of operational loads) and registration of impact damage. In the case of the former, the use of the well-established sensors, such as accelerometers and strain gauges, is limited due to extensive wiring. That is to say, only a few points on the structure can be monitored, which, usually, is not enough [5].

These devices are also sensitive to lightning, current leakage and corrosion [5; 9]. However, a single optical fibre can house even hundreds of sensors thanks to the amazing multiplexing ability [5]. FBG sensor array is also used for damage detection as shown in Figure 3. A smart material with embedded multiple FBG sensors has been developed to monitor the impact damage induced delamination between the face-sheet and the honeycomb core of the sandwich composite parts [9]. 


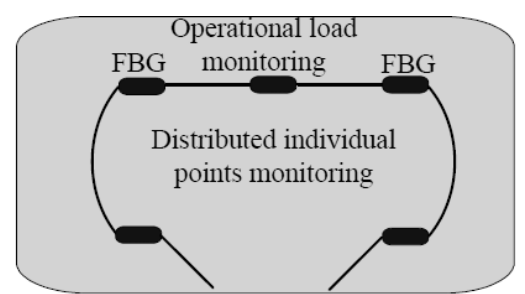

(a)

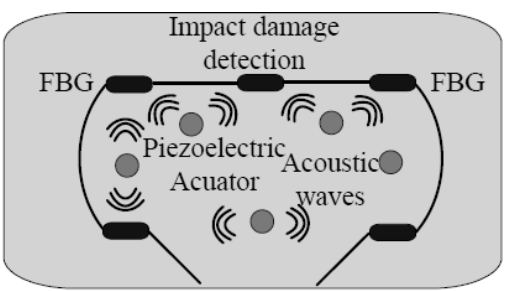

(b)

Fig. 3. SHM of aircraft: a - several FBG sensors to monitoring operational loads; $b$ - hybrid system involving FBG sensors and piezoelectric actuators for detection of impact damages [5]

In [9] researchers made 14 honeycomb core sandwich specimens of rectangular shape with carbon fibre face sheets. The FBG sensor was laid on between the plies of the carbon fibre. Each specimen was cured in a controlled environment. After the embedment the peak wavelength shift generally was less than $1 \mathrm{~nm}$, meaning no significant functional disruptions were introduced.

\section{Composite plates in a controlled environment}

In [4], several FBG sensors were mounted on top of the composite plate and the Lamb wave excitation was performed by piezoelectric actuator discs. A tuneable laser was used for optical fibre illumination and a photodetector was used to detect the optical signal. Sensors were oriented under different angles, since in [6] the directional sensitivity of FBG sensors was demonstrated. Only vibrations in the longitudinal direction to the fibre can be picked up.The damage localization algorithm was based on the time of flight (ToF) with respect to the actuation signal of the highest peaks in the signals after the signal-band filter was applied. From the experimentally determined dispersion curve, a range of propagation velocities for the $S_{0}$ wave was selected corresponding to a Hann windowed 5-peak sinusoid with the selected actuation frequency of $325 \mathrm{kHz}$. With the ToF and propagation velocities, the travelled distances were calculated. It was argued that each distance corresponds to the travelled distance of the excited $S_{0}$ wave between the actuator and damage, plus the distance travelled by the damage reflection, between damage and the FBG considered, as shown in Figure 4.

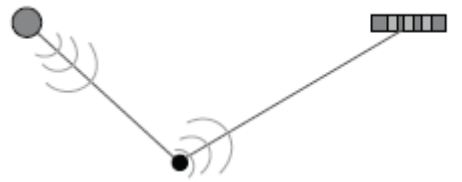

(a)

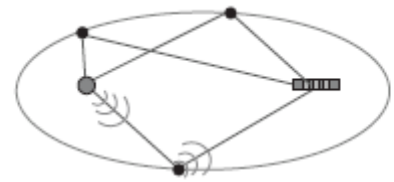

(b)

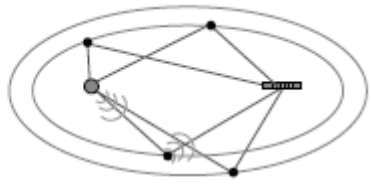

(c)

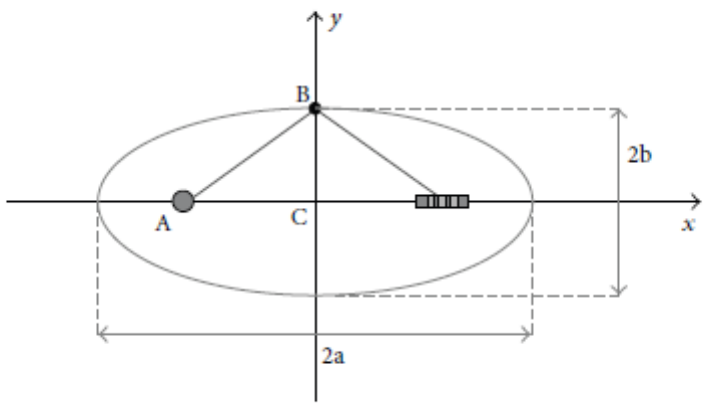

(d)

Fig. 4. Illustration of constructing ellipses: $a-$ one possible location of damage; $b$ - all possible

locations of damage, construction of one ellipse; $\mathrm{c}$ - procedure repeated for both fixed velocities, construction of a ring for one combination of PZT and FBG, and (d) important variables [4]

The total travelled distances for both velocities are constant and can be related to the characteristics of an ellipse (the sum of the distances between any point in an ellipse and its two focal points is constant). Therefore, for each actuator-sensor pair, all the possible damage locations are defined by an ellipse with focal points at the actuator and sensor locations. When the process of ellipse construction was repeated for both velocities, a "ring" representing all possible damage locations was 
obtained for each actuator/sensor pair, as shown in Figure 4 (c). This process was repeated for all the actuator-sensor pairs; the probable damage location on the plate would be associated with the area, where most of the rings intersected [4].

In [6] the researchers proposed a hybrid damage identification system as shown in Figure 5.

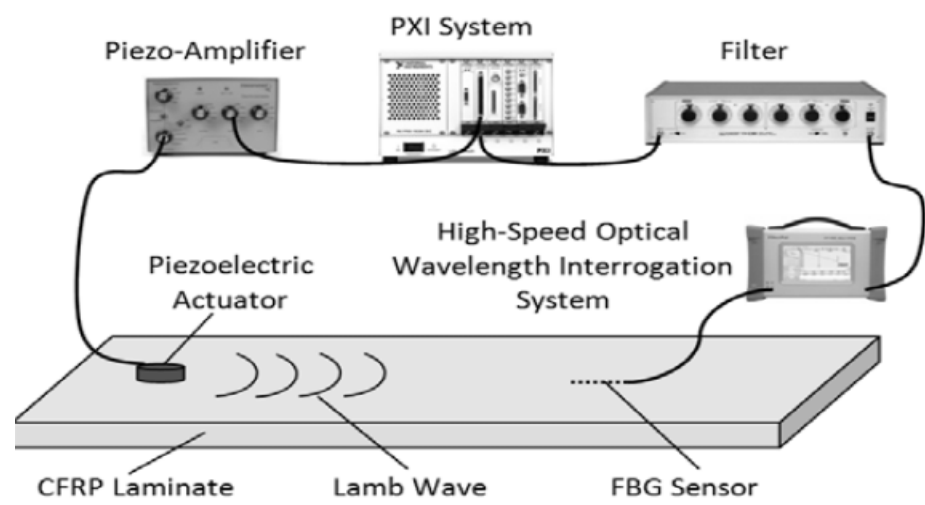

Fig. 5.Hybrid solution for structural damage interrogation - use of both, FBG sensors and piezoelectric actuator [6]

The shift of Bragg wavelength due to damage is registered with a high speed optical wavelength interrogation system. The noise filter is applied to the guided ultrasonic waves to increase the usually low signal-to-noise ratio. These filtered waves are picked up by PXI-6115 system [6]. In [6], the pristine CFRP laminate samples were treated as reference. The delamination damage was associated with a lot of noise due to the fact that the energy of the guided wave is decreased, when passing through the delamination. Hence, for more severe delamination the amplitude of the transmitted waves decreases. There is also a superposition of neighbouring wave packages. The common signal processing techniques include signal averaging, filtering with a band pass filter $[4 ; 6]$ and a subsequent Hilbert transform in order to extract the envelope of the filtered signal. This envelope was used for the extraction of the damage-sensitive features of CFRP laminates.

\section{SHM technologies for pavement and bridge structures}

To ensure high safety of civil infrastructures, such as pavement and bridge structures, assessment of structural integrity in relation to the load-carrying capacity, which decreases due to aging, deterioration, or damage, must be carried out continuously. This structural health monitoring (SHM) is traditionally carried out by periodic visual investigation, discrete electrical or mechanical sensors. However, such sensor deployment is time consuming and they are difficult to install during construction and repairs. They require a large number of electrical connections, complex cabling, they have high susceptibility to electromagnetic interference (EMI), humidity and relatively short lifetime $[10 ; 11]$.

By introducing innovative FOS in a real-time pavement and bridge monitoring system physical parameters, such as strain, temperature and water level condition, can be obtained for the required section in long term [12]. These data can aid structural evaluation, performance assessment and accurate analysis of pavement and bridge structures.

Failure in civil engineering structures occurs, when internal stresses exceed their ultimate limit strength, but, when structures are exposed to cyclic loading, failure can occur due to fatigue - this phenomenon can be observed in structures such as pavements and bridges [13]. In order to design these structures against fatigue and predict the accumulation of distress (amount of cracks, rutting, etc.), there is consecutive monitoring required.

Advanced fibre optic sensing (FOS) technologies have been developing rapidly and in recent years have been applied to the SHM of smart roadways [14]. Experimental results of FBG sensor used have shown that measurement errors of 6 micro strains and 0.13 Celsius for strain and temperature could be achieved, respectively [12].

Strain and temperature real time measurements are also of great interest for the bridge structures. In the work, where the effect of temperature variation on the structural response of the Austrian "ÖBB 
Bruke Großhaslau" bridge has been studied, several parametric graphics proved very useful in identifying temperature as the driver of frequencies changes and not train crossings [15].

Even it is recommended to introduce pavement of bridge real-time monitoring system assisting the structural health monitoring (SHM) and evaluation of distress accumulation in advance, very often SHM is only applied after some pavement or bridge fatigue problems are observed. To prevent sudden failure of the highway system, real-time monitoring of the system was recommended on the road, after 15 years of severely damaged pavement -FBG based sensor assembly was proposed in various layers of the highway pavement system to monitor the 3D strain distribution and assess its various influencing factors for road deterioration monitoring (Figure 6) [16].

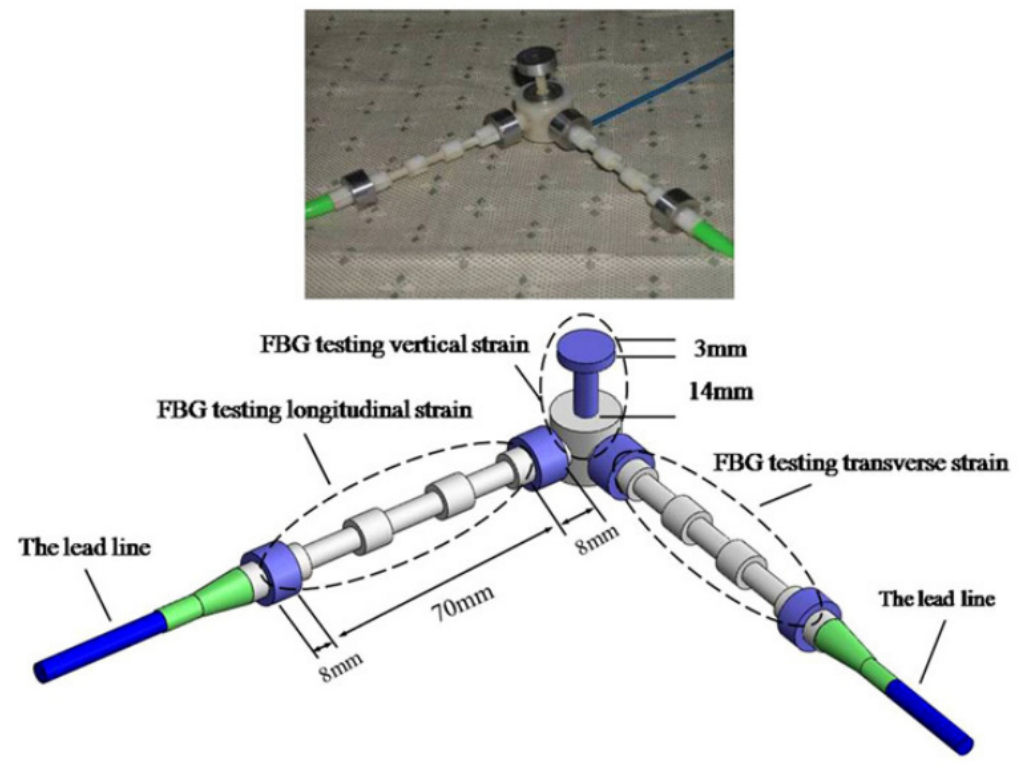

Fig. 6. 3-dimentional strain sensor system [17]

Even there were found reasonably high deviations between in-situ measurements and mathematically simulated with the finite element method, this 3-dymentionl strain measurement system and can eventually be employed in sensing for intelligent vehicle highway systems and health monitoring systems for civil structures, such as bridges [16].

In another study [17], the FBG sensors were used by embedding optical fibres onto a geotextile fabric TenCate GeoDetect ${ }^{\circledR}$ that enhances the performance of the fibre optics sensors, when applied in contact with soil, concrete or asphalt: the geotextile fabric creates an excellent anchoring interface with the surrounding media.

The Mechanical - Empirical Pavement Design Guide [18] design process involves an initial trial design, which is refined through iteration until the predicted distresses meet the design requirements. Real-time structural health monitoring for pavement and bridge structures not only allows to measure physical parameters, but also to calibrate the structural response and distress prediction models [19].

\section{Acknowledgements}

This work has been supported by the European Regional Development Fund Project No. 1.1.1.1/16/A/072.

\section{References}

[1] Werneck M.M., Allil R.C.S. B., Ribeiro B.A. etc.Current Trends in Short- and Long-period Fibre Gratings. INTECH open: Chapter 1 A Guide to Fibre Bragg Grating Sensors, 2013, pp. 1-24.

[2] Thévenaz L., Soto M.A.Novel concepts and recent progress in distributed optical fibre sensing. Proceedings of International conference"Optical Fibre Communications Conference and Exhibition (OFC)", 2016, Anaheim, California, USA, pp. 1-3.

[3] Kashyap R.Fibre Bragg Gratings. Second Edition (Optics and Photonics Series). Academic Press, 2009, pp. 632. 
[4] Barazanchy D., Martinez M., Rocha B. etc. A Hybrid Structural Health Monitoring System for the Detection and Localization of Damage in Composite Structures. Journal of Sensors,Article ID 109403, 2014, pp. 1-10.

[5] Guo H., Xiao G., Mrad N. etc. Fibre Optic Sensors for Structural Health Monitoring of Air Platforms, a Review. Sensors, vol. 11, 2011, pp. 3687-3705.

[6] Li F., Murayama H., Kageyama K. etc. Guided Wave and Damage Detection in Composite Laminates Using Different Fibre Optic Sensors. Sensors, vol. 9, 2009, pp. 4005-4021.

[7] Kinet D., Mégret P., Goossen K.W. etc. Fibre Bragg Grating Sensors toward Structural Health Monitoring in Composite Materials: Challenges and Solutions. Sensors, vol. 14, 2014, pp. 7394-7419.

[8] Cheng-Yu H., Yi-Fan Z., Meng-Xi Z. etc. Application of FBG sensors for geotechnical health monitoring, a review of sensor design, implementation methods and packaging techniques. Sensors and Actuators A, vol. 244, 2016,pp. 184-197.

[9] Ramly R.,Mohd W.K.,Rahman M.K.A. Using Embedded Fibre Bragg Grating (FBG) Sensors in Smart Aircraft Structure Materials. Procedia Engineering, vol. 41, 2012, pp. 600 - 606.

[10]Zhang Z., Huang Y., Palek L. etc. Glass fibre-reinforced polymer-packaged fibre Bragg grating sensors for ultra-thin unbonded concrete overlay monitoring. Structural Health Monitoring, vol. 14(1), 2015, pp. 110-123.

[11] Gebremichael Y.M., Li W., Meggitt B.T. etc. A field deployable, multiplexed Bragg grating sensor system used in an extensive highway bridge monitoring evaluation tests. IEEE Sensors Journal, vol. 5(3), 2005, pp. 510-519, June 2005.

[12] Wang J.-N., Tang J.-L.Optical fibre grating-based sensing system for use in pavement health monitoring, Proceedings of SPIE 6932, "Sensors and Smart Structures Technologies for Civil, Mechanical, and Aerospace Systems", March 9-13, 2008, San Diego, California, USA, pp. 1-10.

[13] Rabaiotti C. Pavement service life prediction and inverse analysis with PLAXIS3D, PLAXIS Bull., vol. 37, 2015, pp. 14-17.

[14] Virginia Department of Transportation, "Virginia's Smart Road Project," VDOT, 2006. [online][19.03.2018]Available at: http://www.virginiadot.org/projects/constsal-smartrd.asp.

[15] Faravelli L., Bortoluzzi D., Messervey T.B. etc.Temperature Effects On The Response Of The Bridge "ÖBB Brucke Großhaslau", Springer Vienna, Vienna, 2014, pp. 85-94.Available at:https://doi.org/10.1007/978-3-7091-1571-8_10.

[16]Zhou Z., Liu W., Huang Y. etc.Optical fibre Bragg grating sensor assembly for 3D strain monitoring and its case study in highway pavement. Mechanical Systems and Signal Processing, vol. 28, 2012, pp. 36-49.

[17] Artières O., Bacchi M., Bianchini P. etc. Strain Measurement in Pavements with a Fibre Optics Sensor Enabled Geotextile. Proceedings of International conference "7th RILEM International Conference on Cracking in Pavements", March 2012, Dordrecht, Netherlands, pp. 201-210.

[18] ARA Inc., Guide for the Mechanistic-Empirical Design of New and Rehabilitated Pavement Structures, Final report, NCHRP 1-37A. Transportation Research Board of the National Academies, Washington, D.C., USA, 2004.

[19] Sias J., Lowe J., Steele M. Local Calibration of the MEPDG for New Hampshire, University of New Hampshire, 2013. [online][19.03.2018] Available at:

https://www.nh.gov/dot/org/projectdevelopment/materials/research/projects/14282s.htm. 